\title{
AN EXTREMAL PROBLEM FOR POLYNOMIALS
}

\author{
A. EREMENKO AND L. LEMPERT
}

(Communicated by Albert Baernstein II)

Dedicated to Paul Erdös on his 80th Anniversary

\begin{abstract}
Let $f(z)=z^{n}+\cdots$ be a polynomial such that the level set $E=$ $\{z:|f(z)| \leq 1\}$ is connected. Then $\max \left\{\left|f^{\prime}(z)\right|: z \in E\right\} \leq 2^{(1 / n)-1} n^{2}$, and this estimate is the best possible.
\end{abstract}

The following question was asked in [1, Problem 4.8]. Let $f$ be a polynomial of degree $n$,

$$
f(z) \sim z^{n}, \quad z \rightarrow \infty .
$$

Put $E_{f}=\{z:|f(z)| \leq 1\}$, and assume that $E_{f}$ is connected. Is it true that

$$
\max \left\{\left|f^{\prime}(z)\right|: z \in E_{f}\right\} \leq \frac{1}{2} n^{2} ?
$$

Pommerenke [2] proved this with $e n^{2} / 2$ instead of $n^{2} / 2$. In a recent survey of unsolved problems [3] Erdős noticed that the estimate (2) has to be replaced by $\frac{1}{2}\{1+o(1)\} n^{2}$ and suggested that Chebyshev polynomials should be extremal.

We will give an affirmative answer to this question. Let us first describe in detail the extremal polynomials for the problem. Define the Chebyshev polynomials $T_{n}, n=1,2, \ldots$, by the usual relation $\cos n z=T_{n}(\cos z)$, and set $f_{n}(z)=T_{n}\left(2^{(1-n) / n} z+1\right)$. Then the polynomials $f_{n}$ have the following properties:

(i) $f_{n}(0)=1$

(ii) $f_{n}^{\prime}(0)=2^{(1 / n)-1} n^{2}$.

(iii) $f_{n}$ is real and all its zeros are negative.

(iv) The critical values of $f_{n}$ are equal to \pm 1 .

The polynomials $f_{n}$ are uniquely characterized by properties (1), (i), (iii), and (iv). To prove this assume that a polynomial $f$ of degree $n$ satisfies (1), (i), (iii), and (iv). Then all zeros of $f$ and $f^{\prime}$ are real and simple. There are exactly $n-1$ points $z<0$ such that $f(z)= \pm 1$ and $f^{\prime}(z)=0$. Choose real constants $a$ and $b$ such that the polynomial $g(z)=f(a z+b)$ has all its zeros (hence critical points) on $(-1,1), g(1)=1$, and $g(-1)=(-1)^{n}$. Then the

Received by the editors November 18, 1992 and, in revised form, December 10, 1992.

1991 Mathematics Subject Classification. Primary 30C10, 30A10.

Key words and phrases. Polynomial, Chebyshev polynomials.

The first author was supported by NSF grant DMS-9101798. The second author was supported by NSF grant DMS-9102978. 
only poles of the rational function $R=g^{2} /\left(1-g^{2}\right)$ are 1 and -1 , and we have

$$
R(z) \sim-n^{2} z^{-2}, \quad z \rightarrow \infty .
$$

We conclude that $g^{2} /\left(1-g^{2}\right)=n^{2} /\left(1-z^{2}\right)$. The solution of this differential equation satisfying $g(1)=1$ is the Chebyshev polynomial $T_{n}(z)=$ $\cos (n \arccos z)$. Now using (1), (i), and (iii) we get $a=2^{(n-1) / n}$ and $b=$ $-2^{(n-1) / n}$, so $f=f_{n}$.

Observe that the conditions and the conclusion of the conjecture are invariant with respect to the substitution $f(z) \mapsto f(z+c), \quad c \in \mathbf{C}$, so the conjecture follows from

Theorem 1. Let $f$ be a polynomial satisfying (1), $|f(0)|=1$, and $E_{f}$ is connected. Then $\left|f^{\prime}(0)\right| \leq f_{n}(0)=2^{(1 / n)-1} n^{2}$. Equality can occur only for $f(z)=c^{-n} f_{n}(c z),|c|=1$.

Proof. It is evident that an extremal polynomial for the problem does exist. We have to prove only that it is equal to $c^{-n} f_{n}(c z),|c|=1$. Let $f$ be an extremal polynomial. From $|f(0)|=1$ follows

$$
f(z)=\lambda \prod_{k=1}^{n}\left(1-\frac{z}{z_{k}}\right), \quad|\lambda|=1,
$$

and (1) implies that $\prod\left|z_{k}\right|=1$. Denote

$$
f^{*}(z)=\prod_{k=1}^{n}\left(1+\frac{z}{\left|z_{k}\right|}\right)
$$

Then $f^{*}$ satisfies $(1)$ and $f^{*}(0)=1$. The condition that $E_{f}$ is connected implies

$$
m(r, f):=\min \{|f(z)|:|z|=r\} \leq 1, \quad 0 \leq r \leq \max _{k}\left|z_{k}\right| .
$$

It is clear that $\left|f^{*}(-r)\right| \leq m(r, f), r \geq 0$, because $|1-r /| z_{k}|| \leq\left|1-r e^{i \theta} / z_{k}\right|$ for all $k$ and $\theta$, so

$$
\left|f^{*}(-r)\right| \leq 1, \quad 0 \leq r \leq \max _{k}\left|z_{k}\right|
$$

Thus the zeros of $f^{*}$ can be connected within $E_{f^{*}}$. The minimum principle therefore implies that $E_{f^{*}}$ is connected. Thus $f^{*}$ satisfies the conditions of the theorem. We have

$$
\left(f^{*}\right)^{\prime}(0)=\sum_{k=1}^{n} \frac{1}{\left|z_{k}\right|} \geq\left|\sum_{k=1}^{n} \frac{1}{z_{k}}\right|=\left|f^{\prime}(0)\right|,
$$

and equality holds if and only if all $z_{k}$ have the same argument. So $f^{*}$ is also extremal, and $f(z)=c^{-n} f^{*}(c z),|c|=1$.

It remains to prove that $f^{*}=f_{n}$. By Rolle's theorem all critical points of $f^{*}$ lie on the segment $[-A,-a]$, where $A=\max \left|z_{k}\right|$ and $a=\min \left|z_{k}\right|$. By (3) all critical values of $f^{*}$ are between 1 and -1 . Denote by $0>a_{1}>a_{2}>\cdots>a_{m}$ all critical points where critical values are equal to \pm 1 . If $m=n-1$ then $f^{*}=f_{n}$ and we are done. 
Now assume that $m \leq n-2$. Put $a_{0}=0$, and construct a real polynomial $p$ of degree at most $m$ such that

$$
p\left(a_{k}\right) f^{*}\left(a_{k}\right)>0, \quad 0 \leq k \leq m .
$$

To do this it is enough to place one zero of $p$ between $a_{k}$ and $a_{k+1}$ for each $k$ such that $f^{*}\left(a_{k}\right)$ and $f^{*}\left(a_{k+1}\right)$ have opposite signs. The sequence $a_{0}, \cdots, a_{m}$ has at most $m$ changes of sign, so the degree of $p$ is at most $m$. For a small number $\varepsilon>0$ consider the polynomial

$$
P(z)=f^{*}(z)+\varepsilon z p(z),
$$

which satisfies (1) and $P(0)=1$. We prove that the set $E_{P}$ is connected if $\varepsilon$ is small enough. First we have $|P(z)| \leq 1,-A \leq z \leq 0$, for small $\varepsilon$ in view of (4) with $1 \leq k \leq m$. Second, denote by $N$ a complex neighborhood of zeros of $f^{*}$, such that $\left|f^{*}(z)\right|<\frac{1}{2}, z \in N$. If $\varepsilon$ is small enough then all zeros of $P$ belong to $N$ and also $|P(z)|<1, z \in N$. So we have $|P(z)| \leq 1$ when $z$ belongs to the connected set $N \cup[-A,-a]$, containing all zeros of $P$. This implies connectedness of $E_{P}$.

So $P$ satisfies all conditions of the theorem. On the other hand, (4) with $k=0$ implies that $P^{\prime}(0)>\left(f^{*}\right)^{\prime}(0)$, which contradicts the extremality of $f^{*}$. This proves the theorem.

\section{REFERENCES}

1. W. K. Hayman, Research problems in function theory, Athlone Press, London, 1967.

2. Christian Pommerenke, On the derivative of a polynomial, Michigan Math. J. 6 (1959), 373-375.

3. Paul Erdös, Some of my favorite unsolved problems, A Tribute to Paul Erdös (A. Baker, B. Bollobás, and A. Hajnal, eds.), Cambridge Univ. Press, Cambridge, 1990.

Department of Mathematics, Purdue University, West Lafayette, Indiana 47907

E-mail address: eremenko@math. purdue.edu

E-mail address: lempert @math.purdue.edu 